\title{
Additively manufactured 4D human head model with tongue movement
} 141

\section{Ewald Unger, Gunpreet Oberoi, Ivo Rausch, Francesco Moscato}

\section{Abstract}

4D printing is the process by which a 3D printed object converts itself into a different object on application of external energy stimulus. This paves the way for spatial self-bending actuation in customdesigned soft structures. Our study is a pioneer translation of this phenomenon in dentistry.

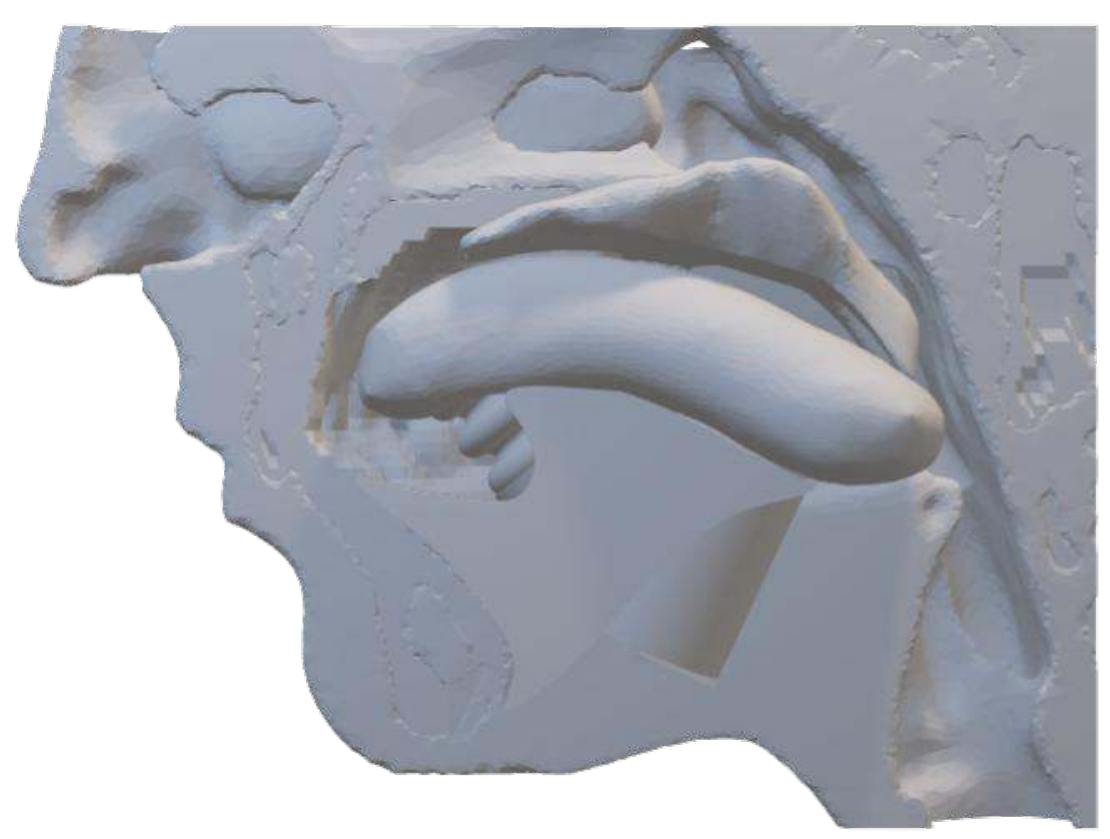

Fig. 1. 4D human tongue model

\section{Background and Aim}

Ethics is an essential element for conducting clinical and pre-clinical research. In this regard, additive manufacturing colloquially called 3D printing has emerged as a promising approach to decrease the load on animal and human trials. With the addition of the fourth dimension (4D) to $3 D$ printing, it is possible to combine anatomical reconstruction with specific physiologic and pathologic activity like muscle movement [1]. In this research, we designed a 4D human tongue model that could act as a platform to test the resistance, retention and efficiency of various velopharyngeal prostheses, palatal obturators and educational purpose.

\section{Methods and Materials}

Results
A 3D model was rendered and segmented using computer software (USL Mimics, Materialise) based on an existing CT and MRI DICOM dataset (patient age-11 years, female) (Fig. 2). The tongue and its inferior muscles were designed with an inbuilt controllable pressure chamber and an adjustable pneumatic source using the same software (Fig. 3). This constituted the functional part (4D) for the tongue. Movement specific composition of 3D printing materials was selected to resemble the applied pressure by the tongue against the palate during swallowing. The model was printed in multi-material polyjet printer. The efficiency of tongue movement of the reconstructed 4D skull model was analyzed qualitatively and quantitatively, by recording the pressure equivalent sensor signals between the palate and tongue [2-3].

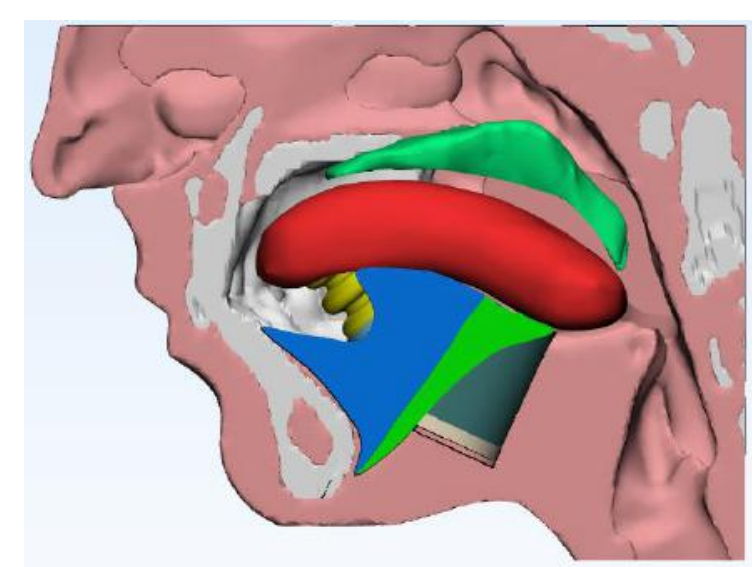

Fig. 2. Tongue position at rest

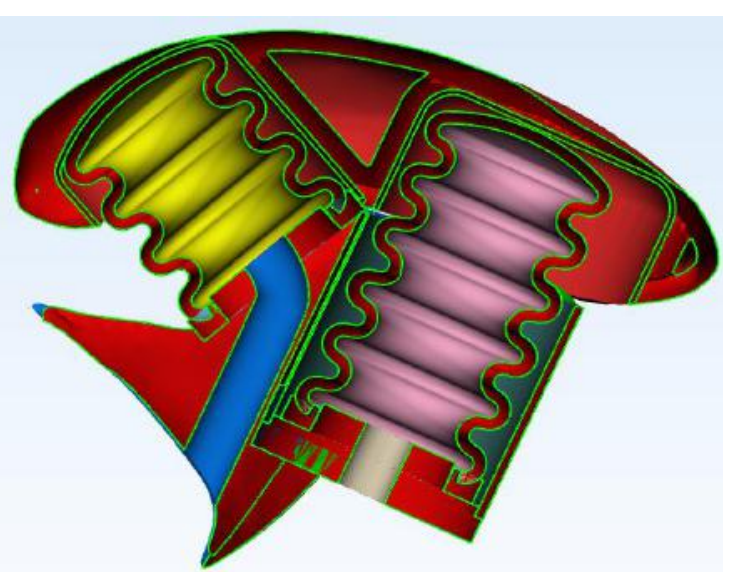

Fig. 3. Biomechanical internal tongue design for 4D model

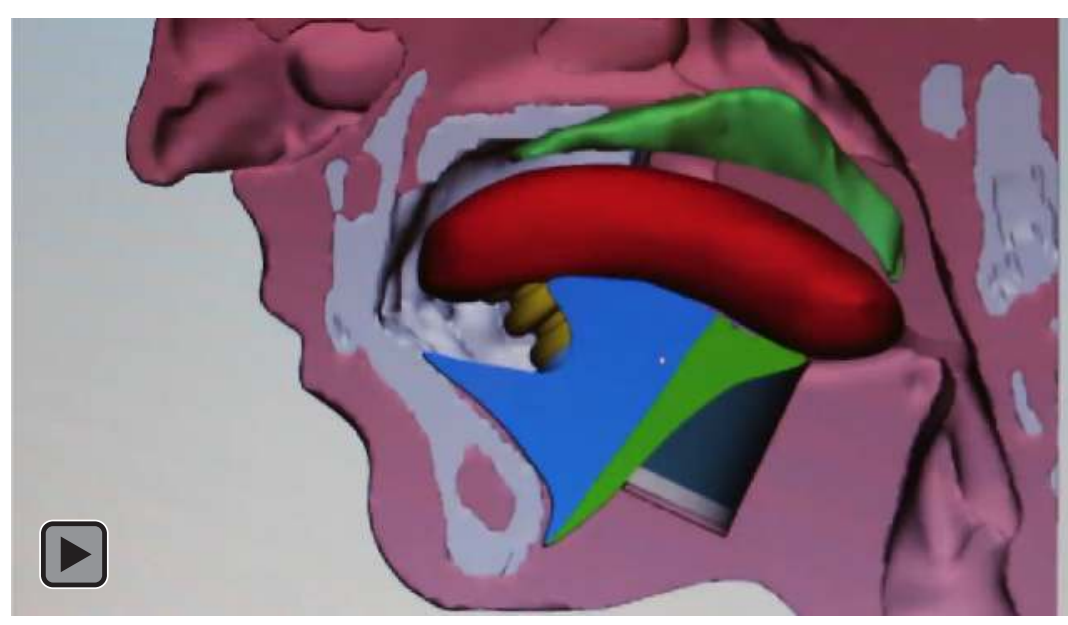

Video 1. Digital model showing tongue movement
With this methodology it was possible to create different physical properties during the production process by mixing the predefined materials from three selected UV light-cured hard and soft materials (Fig. 4). The resulting analysis of movement and pressure showed a good match to the already documented movement and palatal pressure by the tongue during swallowing (Video 3). The positive result of the functional verification allowed us to place various obturator designs in our 4D skull model and test their efficacy in closing the oropharynx during swallowing and other pressure sequences.

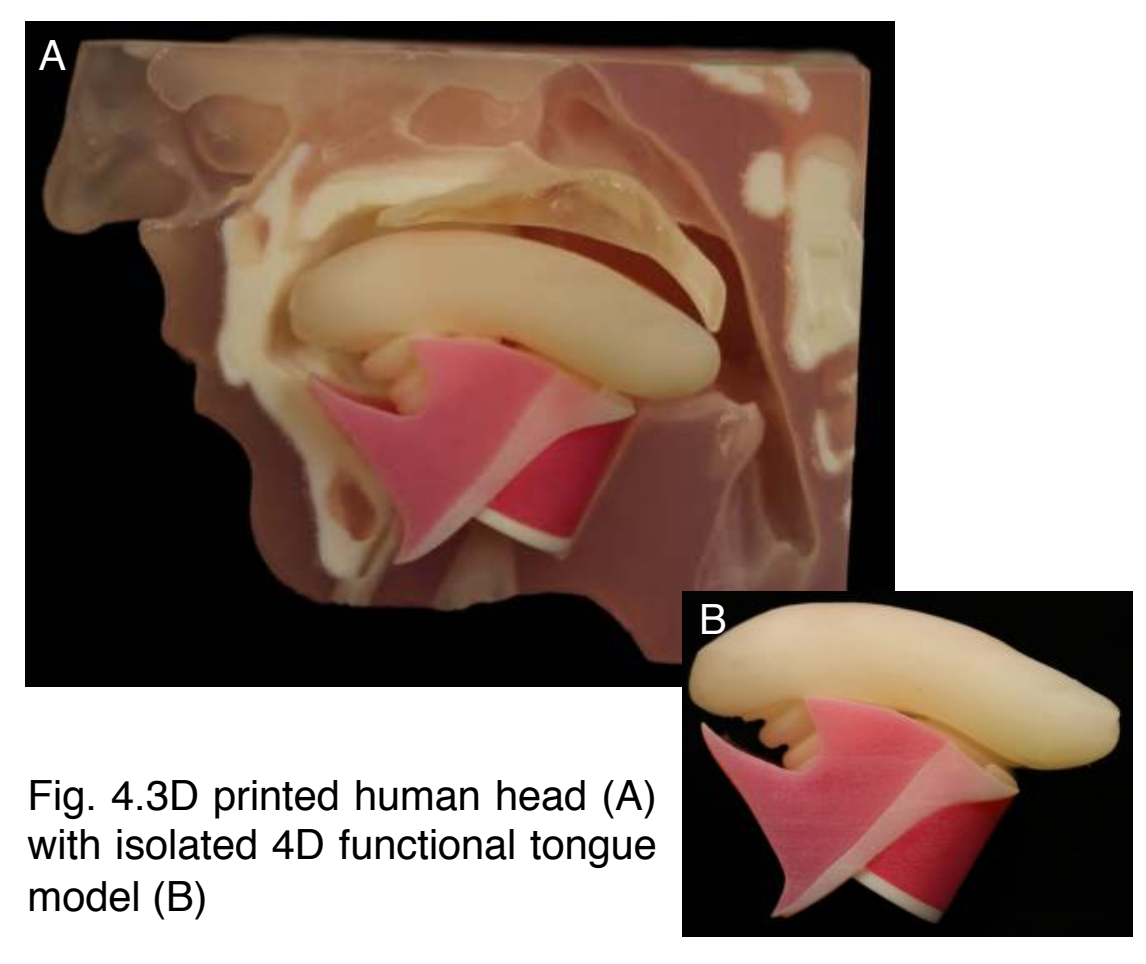

\section{Conclusion}

Our 4D model is very beneficial for verifying multidisciplinary oral prostheses function. The reconstruction workflow offers to create patient specific 4D models to test different palatal and speech defect prostheses. Furthermore, the unique 3D skull model with a 4D functional tongue can be included into clinical training and patient education for better proprioception.

\section{References}

[1]Trenfield SJ, Awad A, Madla CM, Hatton GB, Firth J, Goyanes A, Gaisford $S$, Basit AW. Shaping the future: recent advances of $3 D$ printing in drug delivery and healthcare. Expert Opin Drug Deliv. 2019 Oct;16(10):1081-1094. doi. 10.1080/17425247.2019.1660318. Epub 2019 Sep 3.

[2]Fujiwara, S., Fujiu-Kurachi, M., Hori, K., Maeda, Y. and Ono, T. 2018. Tongue Pressure Production and Submental Surface Electromyogram Activities During Tongue-Hold Swallow with Different Holding Positions and Tongue Length. Dysphagia 33(4), pp. 403-413.

[3]Xu, K., Zeng, J. and Xu, T. 2016. Effect of an intraoral appliance on tongue pressure measured by force exerted during swallowing. American Journal of Orthodontics and Dentofacial Orthopedics 149(1), pp. 55-61. 